Article

\title{
Informational Measure of Symmetry vs. Voronoi Entropy and Continuous Measure of Entropy of the Penrose Tiling. Part II of the "Voronoi Entropy vs. Continuous Measure of Symmetry of the Penrose Tiling"
}

Edward Bormashenko ${ }^{1 *}$, Irina Legchenkova ${ }^{1}$, Mark Frenkel $^{1}$, Nir Shvalb ${ }^{2}$, Shraga Shoval ${ }^{3}$

\author{
Chemical Engineering Department, Engineering Faculty, Ariel University, P.O.B. 3, 407000 Ariel, Israel \\ 2 Department of Mechanical Engineering \& Mechatronics, Faculty of Engineering, Ariel University, P.O.B. 3, Ariel \\ 407000, Israel \\ 3 Department of Industrial Engineering and Management, Faculty of Engineering, Ariel University, P.O.B. 3, Ariel \\ * Correspondence: edward@ariel.ac.il
}

Abstract: The notion of the informational measure of symmetry is introduced according to: $\boldsymbol{H}_{\text {sym }}(G)=-\sum_{i=1}^{k} \boldsymbol{P}\left(\boldsymbol{G}_{\boldsymbol{i}}\right) \boldsymbol{l n} \boldsymbol{P}\left(\boldsymbol{G}_{\boldsymbol{i}}\right)$, where $\boldsymbol{P}\left(\boldsymbol{G}_{\boldsymbol{i}}\right)$ is the probability of appearance of the symmetry operation $\boldsymbol{G}_{\boldsymbol{i}}$ within the given 2D pattern. $\boldsymbol{H}_{\boldsymbol{s y m}}(\boldsymbol{G})$ is interpreted as an averaged uncertainty in the presence of symmetry elements from the group $G$ in the given pattern. The informational measure of symmetry of the "ideal" pattern built of identical equilateral triangles is established as $\boldsymbol{H}_{\text {sym }}\left(\boldsymbol{D}_{3}\right)=1.792$. The informational measure of symmetry of the random, completely disordered pattern is zero, $\boldsymbol{H}_{\text {sym }}=\mathbf{0}$. Informational measure of symmetry is calculated for the patterns generated by the P3 Penrose tessellation. Informational measure of symmetry does not correlate neither with the Voronoi entropy of the studied patterns nor with the continuous measure of symmetry of the patterns.

Keywords: Symmetry; Informational Measure; Penrose tiling, Voronoi entropy, continuous symmetry measure; ordering.

\section{Introduction}

The paper introduces the connection between two fundamental physical notions, namely the notion of symmetry and the Shannon measure of information [1-2], abbreviated in the text SMI. For any random discrete variable $\boldsymbol{X}$, characterized by a probability distribution: $\boldsymbol{P}\left(\boldsymbol{X}_{1}\right), \boldsymbol{P}\left(\boldsymbol{X}_{2}\right) \ldots \boldsymbol{P}\left(\boldsymbol{X}_{\boldsymbol{N}}\right)$ the SMI, denoted $\boldsymbol{H}(\boldsymbol{X})$, has been defined as:

$$
H(X)=-\sum_{i=1}^{N} P_{i}\left(X_{i}\right) \log _{2}\left(X_{i}\right)
$$

The value $\boldsymbol{H}(\boldsymbol{X})$ has the same mathematical form as the entropy in statistical mechanics [3-4]. Thus, Claude Shannon called this value, "entropy", which gave rise to numerous and widespread misinterpretations, discussed in detail in refs. 5-9, in which distinction between SMI and a "true" thermodynamic entropy (in other words the "Boltzmann entropy") was treated in detail. We apply SMI, defined by Eq. 1 to the analysis of the 2D patterns, possessing given elements of symmetry. Thus, the information measure of symmetry (IMS) is introduced. A connection between two fundamental concepts of information and symmetry breaking was addressed in ref. 10. Information content of spontaneous symmetry breaking was studied in ref. 11. 
Our paper continues the series of paper, in which the fundamental problem of the connection between ordering and symmetry in physical systems and spatial patterns was addressed [12,13]. The fundamental question is formulated as follows: what is the quantitative measure of ordering? In physics, ordering is quantified by the entropy of the physical system $[3,4,12,13]$. We already suggested that ordering in physical systems may be sometimes identified with symmetrizing of the system: in other words, introducing the elements of symmetry into an initially disordered physical system will necessarily order the system and consequently decrease its entropy [12-13]. A the same time various measures were suggested for quantification of symmetry of physical systems, one of which is the continuous measure of symmetry (abbreviated CSM and denoted $\tilde{\boldsymbol{S}}(\boldsymbol{G})$ for the G-symmetry shape), defined as the sum of minimum squared distances required to move the points of the original shape in order to obtain a symmetrical shape [14-22]. In our recent paper we applied CSM for the analysis of the Penrose tessellations, explaining the rotational symmetry of quasi-crystals [23]. In the present paper we introduce the alternative measure of the symmetry of 2D patterns, namely the informational measure of symmetry (ISM) and apply the suggested measure for the analysis of the patterns generated by the Penrose tiling. We compare the informational measure of symmetry with the Voronoi entropy (denoted $\boldsymbol{S}_{\boldsymbol{v o r}}$ ) [23-35] and the continuous measure of symmetry [14-22] calculated for the patterns generated by the Penrose tessellation P3, presented in Figure 1. A Penrose tiling, presented in Figure 1, is an aperiodic tiling that has a 5-fold rotational and reflection symmetry [36-37]. Penrose tiling supplies the explanation for the physical structure of quasicrystals, demonstrating the 5-fold symmetry [36-37]. The translational symmetry is absent in the Penrose tiling. We analyzed symmetry of the Penrose tiling in parallel with the informational measure of symmetry, Voronoi Entropy and the continuous measure of symmetry.

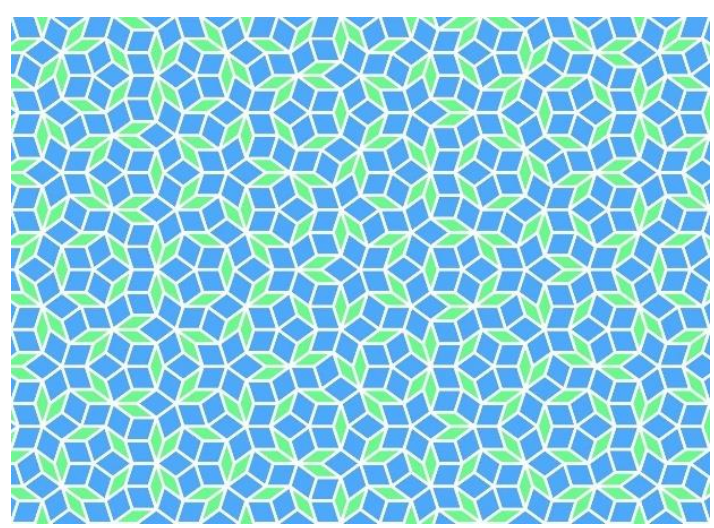

(a)

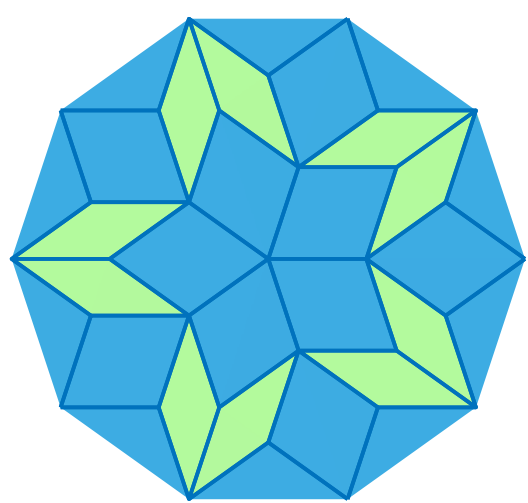

(b)

(c)

Figure 1. (a) The Penrose tiling P3 is depicted. The translation symmetry is absent in the pattern, however, the pattern demonstrates the fivefold rotational symmetry The Penrose tiling P3 demonstrates $H_{\text {sym }}(G)=1.386$.

\section{Materials and Methods}

The MATLAB software was used for calculation of the continuous measure of symmetry of the studied patterns. To create the Voronoi diagrams, we used moduli of the program developed at the Department of Physics and Astronomy at the University of California (Department of Physics and Astronomy University of California, Irvine) (https://www.physics.uci.edu/ foams/do_all.html).

\section{Results and Discussion}

\subsection{Definition of the Informational Measure of Symmetry}

We already demonstrated in our recent research that the Voronoi entropy and the continuous measure of symmetry do not exhaust the quantification of ordering in 2D patterns [23]. We propose now he alternative approach to the problem and introduce the "informational measure of symmetry". Consider the 2D pattern, containing $N$ polygons demonstrating certain elements of symmetry 
(rotational symmetry; centers of symmetry, axes of symmetry, etc.), denoted $\boldsymbol{G}_{\boldsymbol{i}}, \boldsymbol{i}=\mathbf{1}, \mathbf{2} \ldots \boldsymbol{k}$, where $k$ is a number of non-identical symmetry operations. Thus, the informational measure of symmetry will be defined similarly to Eq. 1 as:

$$
H_{\text {sym }}(G)=-\sum_{i=1}^{k} P\left(G_{i}\right) \ln P\left(G_{i}\right)
$$

where $P\left(G_{i}\right)$ is the probability of appearance of the symmetry operation $G_{i}$ within the polygons constituting the pattern, defined as:

$$
P\left(G_{i}\right)=\frac{m\left(G_{i}\right)}{N_{G}} \leq 1
$$

where $N_{G}=\sum_{i=1}^{k} m\left(G_{i}\right)$ is a total number of symmetry elements (operations) appearing in the polygons recognized in a given pattern and $m\left(G_{i}\right)$ is a number of the same symmetry elements (operations) $G_{i}$ calculated for a given pattern. The normalization condition given by Eq. 4 takes place:

$$
\sum_{i=1}^{k} P\left(G_{i}\right)=1
$$

It is noteworthy that the entire 2D pattern may be symmetrical or non-symmetrical; the definition of the informational measure of symmetry is not sensitive to the symmetry of the entire pattern. Consider first an "ideal pattern" built of identical equilateral triangles, depicted in Figure 2 a.

The symmetry group of the equilateral triangle is the dihedral symmetry group $D_{3}$. The symmetry group $D_{n}$ generally comprises $n$ symmetry axes and $n$ rotations, given by the angles $\varphi_{n}=$ $k \frac{2 \pi}{n} ; k=0,1,2 \ldots n-1$. Thus, in the case of equilateral triangles, shown in Figure $2 \mathbf{a}$ we have $N_{g}=2 n p=$ $6 p$ elements of symmetry, where $p$ is the total number of triangles in the pattern. The number of each of us of the elements of symmetry in the addressed pattern equals $m\left(G_{i}\right)=1 \times p$. Thus, probabilities $P\left(G_{i}\right)$ are immediately calculated as $\left(G_{i}\right)=\frac{1 \times p}{(3+3) \times p}=\frac{1}{6}$. Hence, for this pattern the informational measure of symmetry is calculated wit Eq. 4

$$
H_{\text {sym }}\left(D_{3}\right)=-\sum_{i=1}^{6} P\left(G_{i}\right) \ln P\left(G_{i}\right)=\sum_{i=1}^{6} 1 / 6 \ln (1 / 6)=1.792
$$

Obviously, for the same pattern $S_{v o r}(G)=0 ; \hat{S}(G)=0$ take place. Now consider the opposite case of the completely disordered pattern, depicted in Figure $\mathbf{2 b}$. In this case we recognize for all of the polygons constuting the patterm the single element of symmetry, namely the rotation $\varphi_{1}=k \frac{2 \pi}{1}=2 \pi$; thus, $N_{G}=p$; thus, $P\left(G_{1}\right)=1$ and consequently $H_{\text {sym }}=-\sum_{i=1}^{1} P\left(G_{i}\right) \ln P\left(G_{i}\right)=\sum_{i=1}^{6} 1 \ln (1)=0$. 


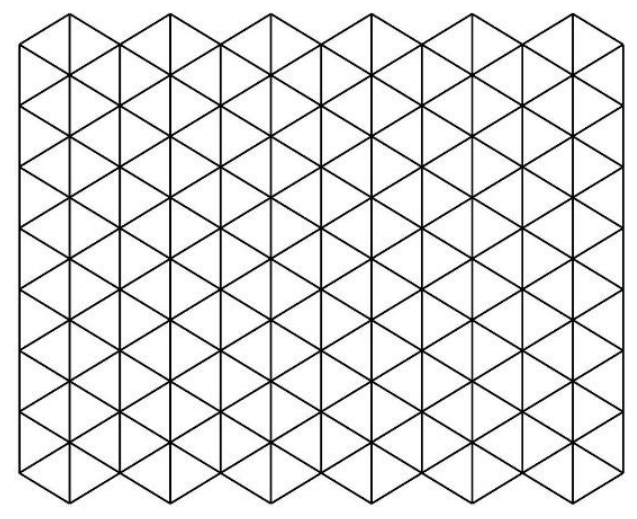

(a)

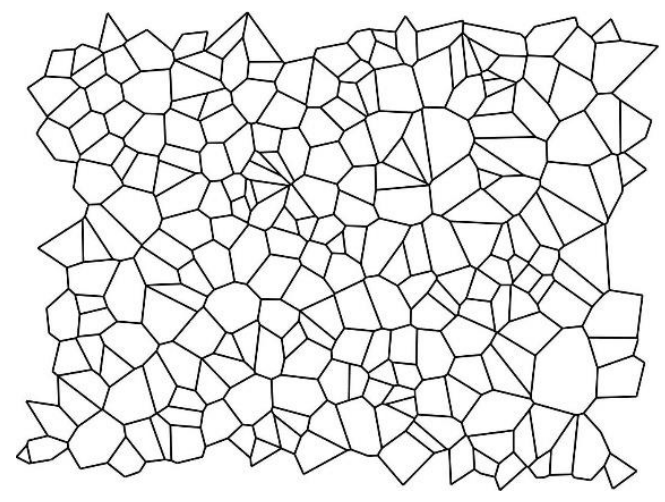

(b)

Figure 2. (a) Pattern (Voronoi tessellation) built of identical equilateral triangles, demonstrating: $H_{\text {sym }}(G)=1.792 ; S_{\text {vor }}=0 ; \hat{S}(G)=0 \%$. Is shown(b) Pattern (Voronoi tessellation) built of different unsymmetrical polygons, demonstrating: $H_{\text {sym }}(G)=0 ; S_{\text {vor }}=1.6103 ; \hat{S}(G)=46.91 \%$ is depicted.

The Voronoi entropy and the continuous measure of symmetry for the same random, disordered pattern shown in Figure 2b equals: $S_{v o r}=1.6103 ; \hat{S}(G)=46.91 \%$, which is close to the Voronoi entropy $S_{v o r}=1.71$ calculated for disordered patterns in refs. 39-40.

\subsection{Informational Measure of symmetry of the Patterns generated by the Penrose Tiling}

A given Penrose tiling generates a number of the Voronoi diagrams, shown in Figures 3(a)-(g) (depicted in the left column of Figure 3). We already introduced in ref. 23 three main types of the Voronoi diagrams, arising from the Penrose tiling P3, namely:

$a$-type Voronoi diagrams (abbreviated $a$-diagrams), where the centers of the Penrose rhombs are taken as the seeds, shown in Figure 3(a);

$b$ - type Voronoi diagrams (abbreviated $b$-diagrams) where the vertices of the rhombs constituting the Penrose tiling, are taken as the seeds, depicted in Figure 3(b);

$c$-type Voronoi diagrams (abbreviated $c$-diagrams) where the centers of the edges of Penrose rhombs are taken as the seeds, shown in Figure 3(c).

In addition, the combinations of the $a, b$ and $c$ diagrams were addressed, denoted $a b, a c, b c$ and $a b c$ correspondingly. These Voronoi diagrams are shown in Figures 3(d)-(g). For example, $a b-$ diagram (depicted in Figure 3(d)) is the Voronoi diagram arising from merging of the seed points appearing in $a$ - and $b$-diagrams. Note, that $a, b, c, a b, a c, b c$ and $a b c$-type Voronoi diagrams possess the same groups of symmetry, reflecting the groups of symmetry of the seed points. In particular, all of the diagrams are characterized by the 5-fold rotational symmetry as well as the mirror plane symmetry which can be recognized from Figure 3. The elements of symmetry appearing in the patterns are cataloged in Table 1. It should be emphasized that in spite of the fact that all of the entire patterns are characterized by the 5 -fold rotational symmetry, patterns $a, c$, and $a c$ are built from the polygons, which do not demonstrate this kind of yje rotational symmetry.

Now address the quantification of symmetry of the patterns depicted in Figure 3 . Which of the patterns are more and which are less symmetric? We'll demonstrate that the answer to this question is far from to be trivial and it is ambigous. The values of the continuous symmetry measure $(\tilde{S}(G)$ and $\hat{S}(G))$ and the Voronoi Entropy $\left(S_{v o r}\right)$ of the patterns were reported in ref. 23. The results of the calculation of the informational measure of symmetry $H_{\text {sym }}(G)$ for seven investigated Penrose tilings are summarized in Figure 3 and Table 2. 
$a$

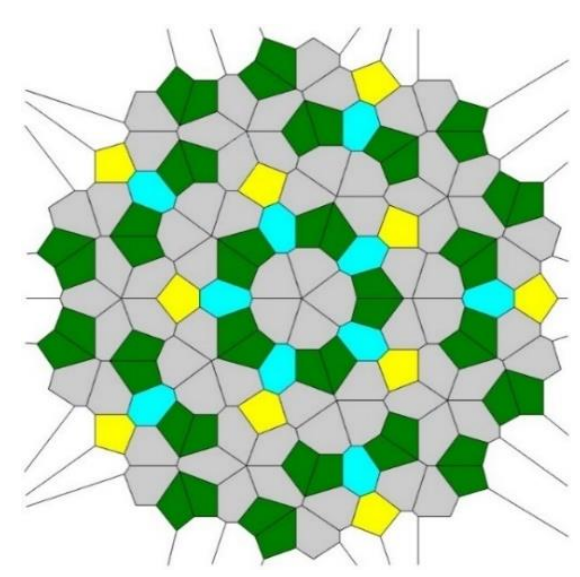

$b$

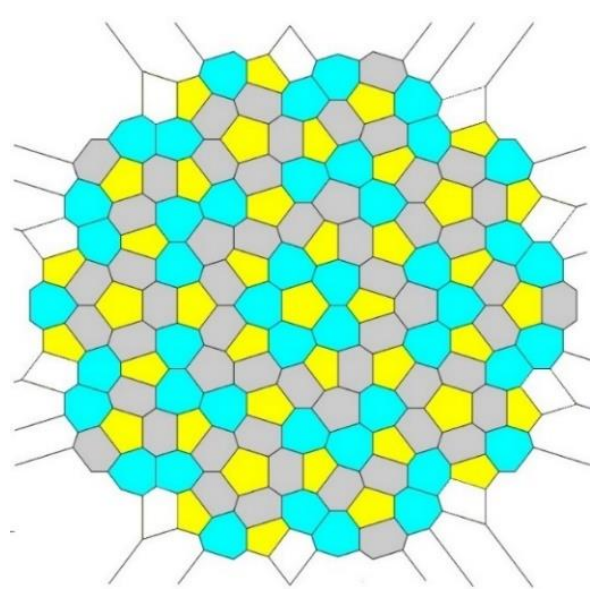

c

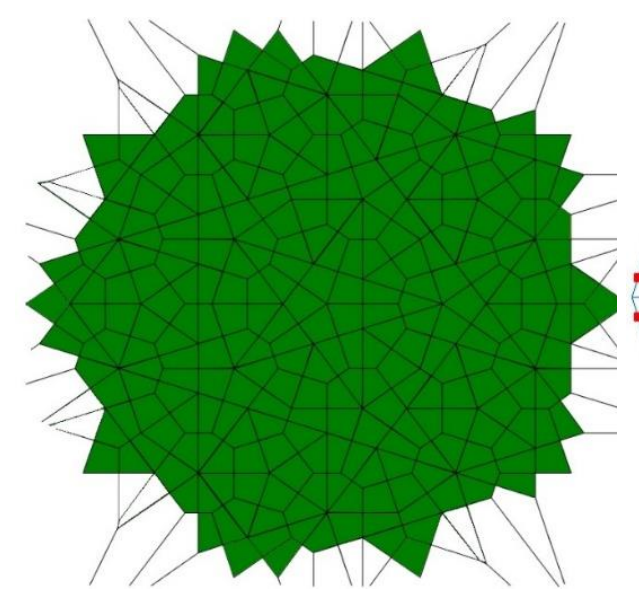

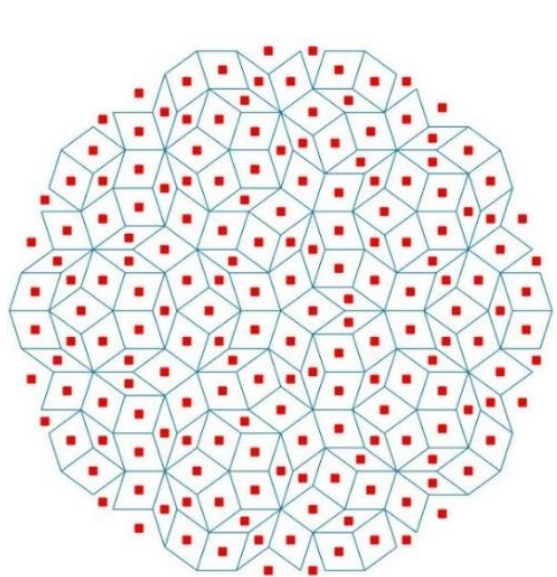

IMS=

$H_{\text {sym }}=$

0.611

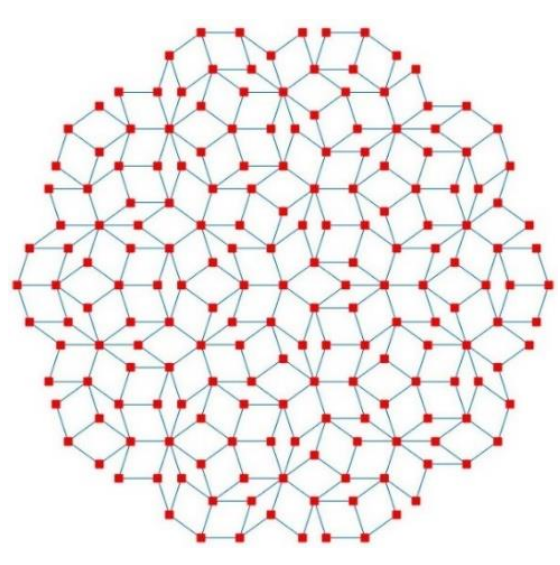

IMS=

$H_{\text {sym }}=$

1.310

IMS=

$H_{\text {sym }}=$

0.569

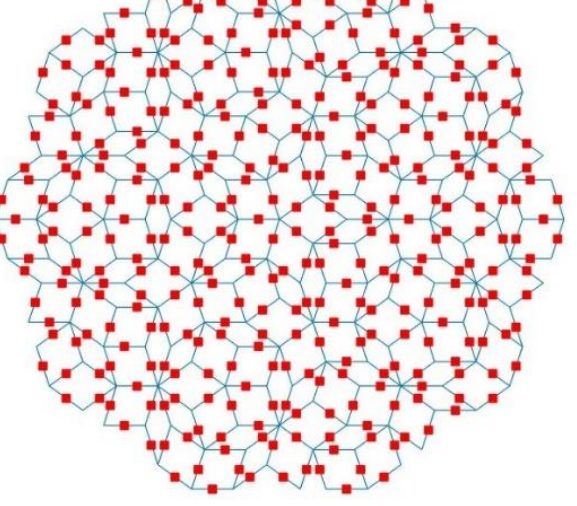



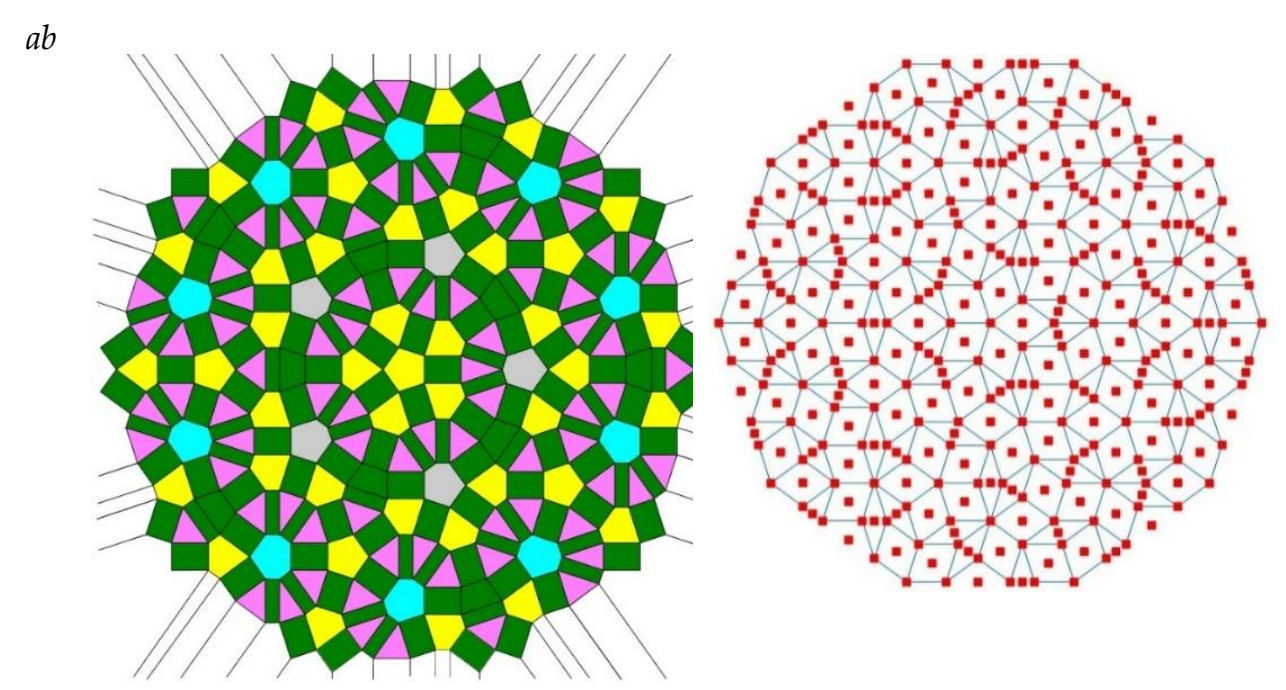

IMS=

$a c$

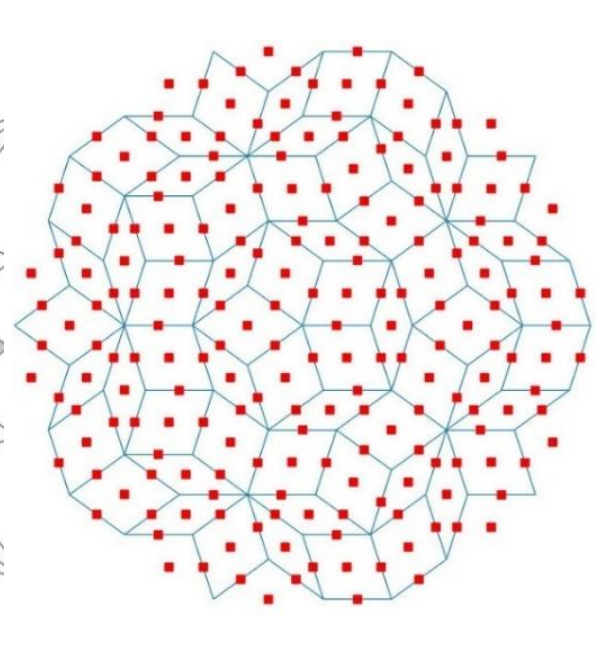

IMS=

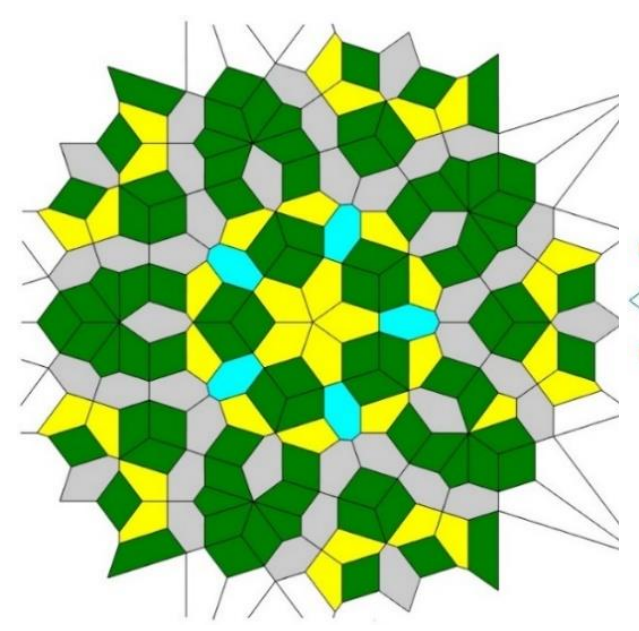

$H_{\text {sym }}=$

1,161

$b c$
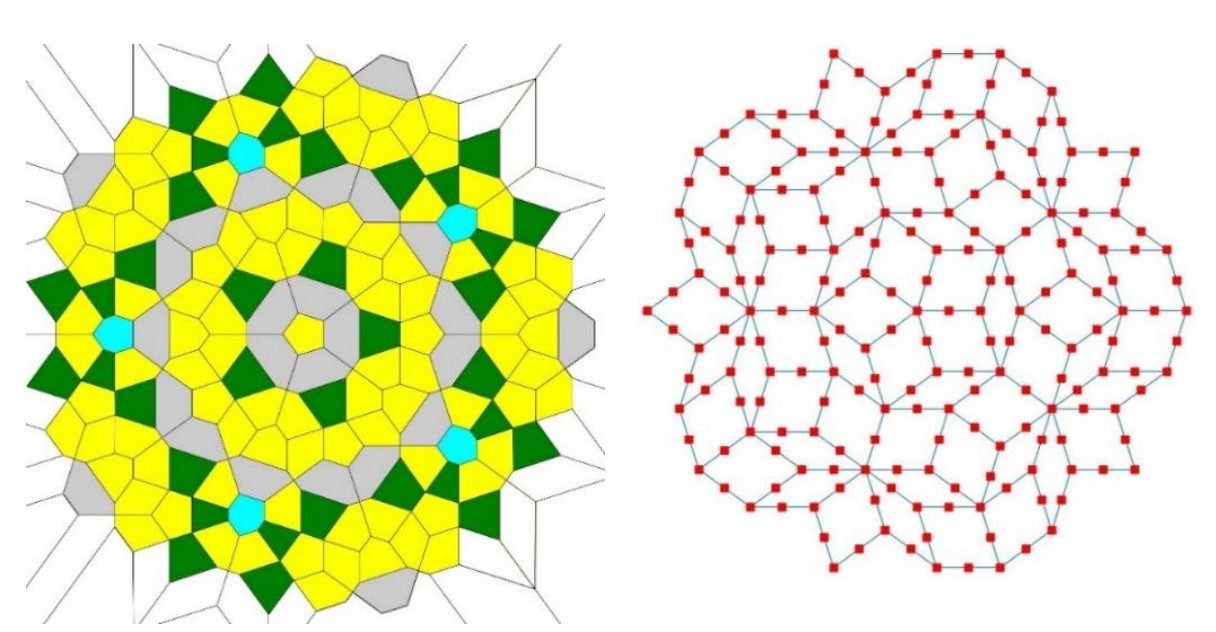

IMS=

$H_{\text {sym }}=$

0.835 


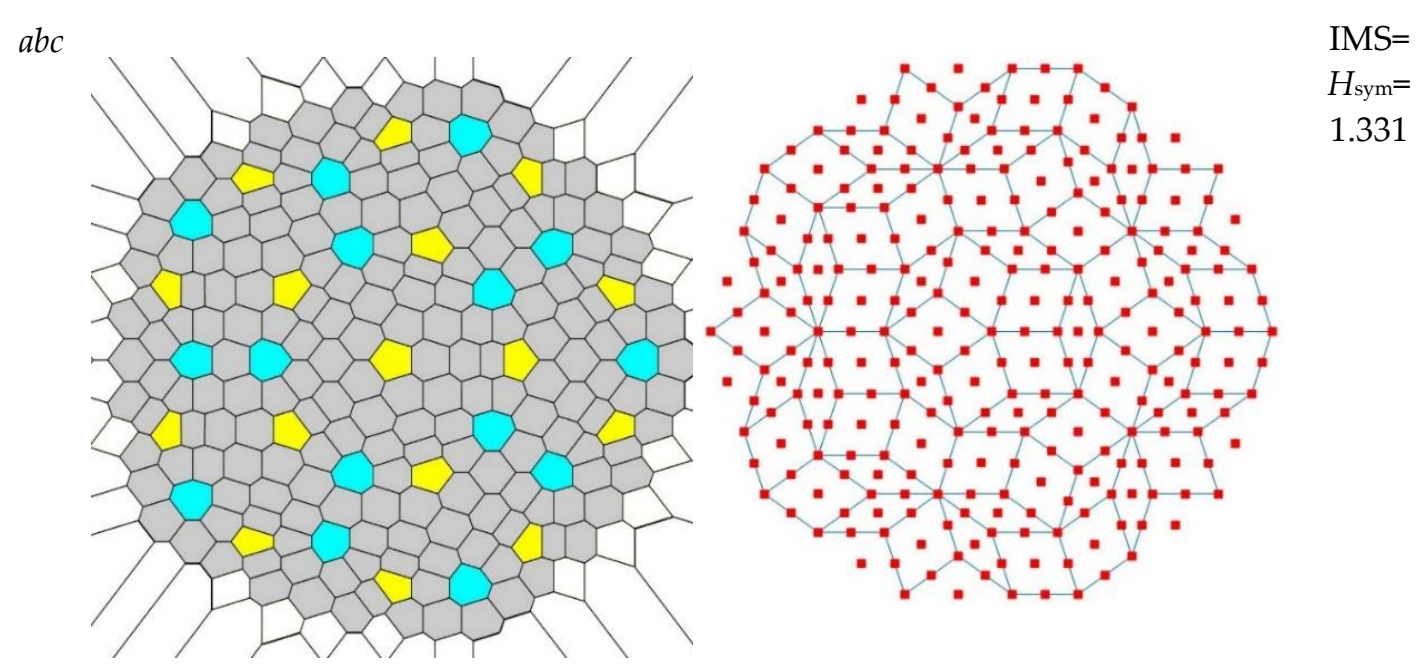

Figure 3. Patterns emerging from the P3 Penrose tiling and their informational measure of symmetry (IMS) calculated with Eq. 2. (a) $a$-type Voronoi tessellation; (b) $b$-type; (c) $c$-type; (d) $a b$-type ( $a$ and $b$-types combined); (e) ac-type; (f) bc-type; (g) abc-type ( $a, b$, and $c$-types combined). Color mapping: magenta polygons are triangles, green are tetragons, yellow are pentagons, grey are hexagons, blue are heptagons.

Table 1. Symmetry elements presented in different types of Voronoi Diagrams generated by P3 Penrose tiling.

\begin{tabular}{ccccccccccccc}
\hline $\begin{array}{c}\text { Diagram } \\
\text { type }\end{array}$ & \multicolumn{4}{c}{ Mirror planes number $\left(m_{p}\right)$} & \multicolumn{5}{c}{ Number of rotation axes $\left(m_{r}\right)$} \\
\hline a & $p_{1}$ & $p_{5,2}$ & $p_{5,3}$ & $p_{5,4}$ & $p_{5,5}$ & $p_{2,2}$ & $(2 \pi)$ & $\left(\frac{2 \pi}{5}\right)$ & $\left(\frac{4 \pi}{5}\right)$ & $\left(\frac{6 \pi}{5}\right)$ & $\left(\frac{8 \pi}{5}\right)$ & $(\pi)$ \\
b & 141 & - & - & - & - & - & 140 & - & - & - & - & - \\
$\mathrm{c}$ & 100 & - & - & - & - & - & 290 & - & - & - & - & - \\
$\mathrm{ab}$ & 306 & 6 & 6 & 6 & 6 & 160 & 311 & 6 & 6 & 6 & 6 & 160 \\
$\mathrm{ac}$ & 65 & - & - & - & - & 35 & 165 & - & - & - & - & 35 \\
$\mathrm{bc}$ & 91 & 1 & 1 & 1 & 1 & - & 161 & 1 & 1 & 1 & 1 & - \\
$\mathrm{abc}$ & 151 & 1 & 1 & 1 & 1 & 60 & 221 & 1 & 1 & 1 & 1 & 60 \\
\hline
\end{tabular}

$m_{r}$ is the total number of rotation axes corresponding to the rotation angle $\varphi_{n}=\frac{2 \pi}{n}$.

$m_{p}$ is the total number of corresponding mirror planes.

$p_{1}$ denotes the single mirror plane.

$p_{n . k}$ denotes $k$-type-mirror planes appearing in the polygon possessing $n$-fold symmetry.

$p_{5.2}$ denotes the second-type mirror planes appearing in the 5-fold symmetric polygon.

Table 2. Informational measure of symmetry (IMS), Voronoi Entropy (VE) and Continuous Measure of Symmetry (CSM) Calculated for the Voronoi Diagrams generated by the Penrose tiling (see Figure 3).

\begin{tabular}{ccccccc}
\hline $\begin{array}{c}\text { Diagram } \\
\text { type }\end{array}$ & $\begin{array}{c}\text { Polygon types } \\
\text { number }\end{array}$ & $\begin{array}{c}\text { IMS, } \\
\boldsymbol{H}_{\text {sym }}\end{array}$ & $\begin{array}{c}\text { Voronoi } \\
\text { Entropy, } \boldsymbol{S}_{\text {vor }}\end{array}$ & $\begin{array}{c}\text { CSM } \\
\tilde{\boldsymbol{S}}(\boldsymbol{G})\end{array}$ & $\begin{array}{c}\text { CSM } \\
\widehat{\boldsymbol{S}}(\boldsymbol{G}) \%\end{array}$ & $\boldsymbol{\Psi}$ \\
\hline$a$ & 4 & 0.611 & 1.1364 & 0.1138 & 33.74 & 5.37 \\
$b$ & 3 & 1.310 & 1.0847 & 0.0367 & 19.15 & 35.70 \\
$c$ & 1 & 0.569 & 0 & 0.1099 & 33.15 & 5.18 \\
$a b$ & 5 & 1.566 & 1.122 & 0.0619 & 24.87 & 25.30 \\
$a c$ & 4 & 1.161 & 1.1026 & 0.0931 & 30.52 & 12.47 \\
$b c$ & 4 & 0.835 & 1.0371 & 0.0912 & 30.2 & 9.16 \\
$a b c$ & 3 & 1.331 & 0.5026 & 0.0515 & 22.7 & 25.84 \\
\hline
\end{tabular}


What is the meaning of the introduced informational measure of symmetry $\boldsymbol{H}_{\text {sym }}(\boldsymbol{G})$ ? In our interpretation we follow the approach developed in refs. 5, 38. Following ref. 5 three pathways of interpretation of $\boldsymbol{H}_{\text {sym }}(\boldsymbol{G})$ are possible, namely:

i) $\quad H_{\text {sym }}(\boldsymbol{G})$ is interpreted as an averaged uncertainty in the presence of symmetry elements from the group $G$ in the given pattern.

ii) $\boldsymbol{H}_{\text {sym }}(\boldsymbol{G})$ may also be understood as a measure of the average unlikelihood, or unexpectedness of presence of symmetry elements constituting group $G$ in the given $2 \mathrm{D}$ pattern.

iii) The most complicated is the information interpretation of the $\boldsymbol{H}_{\boldsymbol{s y m}}(\boldsymbol{G})$. It turns out that the quantity $\boldsymbol{H}_{\text {sym }}(\boldsymbol{G})$, provides us with a measure of this information in terms of the minimum number of questions one needs to ask in order to find the presence of elements of symmetry $\boldsymbol{G}_{\boldsymbol{i}}$ in a given pattern, when $\boldsymbol{P}\left(\boldsymbol{G}_{\boldsymbol{i}}\right)$, i.e. the probabilities of appearance of the symmetry operation $\boldsymbol{G}_{\boldsymbol{i}}$ within the pattern are prescribed. It turns out, that the quantity $\boldsymbol{H}_{\text {sym }}(\boldsymbol{G})$, provides a minimum measure of information needed to describe a given pattern as a composition of $\boldsymbol{G}_{\boldsymbol{i}}$ elements of symmetry [5].

Whatever is the interpretation of the informational measure of symmetry $\boldsymbol{H}_{\boldsymbol{s y m}}(\boldsymbol{G})$ it provides an averaged information about the entire pattern, and it is not related to the specific symmetry operations $\boldsymbol{G}_{\boldsymbol{i}}$, as stressed in ref. 5. Let us start our analysis from the pattern " $a$ ". As it is recognized from Table 2 it is recognized by the relatively low value of $\boldsymbol{H}_{\text {sym }}(\boldsymbol{G})=\mathbf{0 . 6 1 1}$ and the highest for the studied patterns values of $\boldsymbol{S}_{\text {vor }}=\mathbf{1 . 1 3 6 4}$ and $\widetilde{\boldsymbol{S}}(\boldsymbol{G})=\mathbf{0 . 1 1 3 8}(\widehat{\boldsymbol{S}}(\boldsymbol{G})=\mathbf{3 3 . 7 4 \% )}$. How these data should be interpreted? Low values of the informational measure of symmetry $\boldsymbol{H}_{\text {sym }}(\boldsymbol{G})=\mathbf{0 . 6 1 1}$ emerge from the fact that the polygons constituting pattern " $a$ " possess only two elements symmetry, namely $\mathbf{2} \boldsymbol{\pi}$ - rotations (which is an identity element of the symmetry group) and the mirror planes (see Table 1). Thus, the averaged uncertainty in the presence of symmetry elements, quantified by $\boldsymbol{H}_{\text {sym }}(\boldsymbol{G})$ is low. On the other hand, pattern " $a$ " is built from four types of different polygons, depicted in Figure 3a; this results in the relatively high value of the Voronoi entropy. An effort necessary for symmetrization of the pattern " $a$ " is also high; this explains the high value of $\widehat{\boldsymbol{S}}(\boldsymbol{G})$. Thus, we came to the following main conclusions:

i) The quantification of symmetry of the pattern has a "fine structure" and could not be expressed with a single numerical value.

ii) The information measure of symmetry, the Voronoi entropy and the continuous measure of symmetry are not necessarily correlated.

Now address pattern " $b$ ". It is characterized by the high value of the informational measure of symmetry $\boldsymbol{H}_{\text {sym }}(\boldsymbol{G})$, the high value of the Voronoi entropy $\boldsymbol{S}_{\boldsymbol{v o r}}$ and the lowest possible value of the continuous measure of symmetry $\widehat{\boldsymbol{S}}(\boldsymbol{G})$ (see Table 2 ). The high value of $\boldsymbol{H}_{\text {sym }}$ arises from the broad diversity of the symmetry elements appearing in this pattern (see Table 1); the relatively high value of the Voronoi entropy calculated for pattern " $a$ " emerges from the three kinds of polygons constituting this pattern and the low of the continuous measure of symmetry evidences "the low effort" necessary for converting polygons into the perfectly symmetric shapes.

The high values of $\boldsymbol{H}_{\text {sym }}(\boldsymbol{G})$ and $\boldsymbol{S}_{\boldsymbol{v} \text { or }}$ derived for the pattern " $a b$ " are explained in the same way; however, the effort necessary for symmetrization of this pattern and quantified by $\tilde{\boldsymbol{S}}(\boldsymbol{G})$ is twice higher that that established for pattern " $b$ ".

Now address the most paradoxical pattern " $c$ " which is characterized by the lowest possible Voronoi entropy $\boldsymbol{S}_{\boldsymbol{v o r}}=\mathbf{0}$ and the lowest for studied patterns value of the informational measure of symmetry $\boldsymbol{H}_{\text {sym }}=\mathbf{0 . 5 6 9}$. Pattern " $c$ " is built from quadrangles only; thus, the Voronoi entropy of this pattern is zero. Only two elements of symmetry appear in this pattern, namely: the mirror plane and the $\mathbf{2} \boldsymbol{\pi}$ - rotational symmetry (see Table $\mathbf{1}$ ), hence the averaged uncertainty in the presence of symmetry elements is low. This implies the low value of the informational measure of symmetry $\boldsymbol{H}_{\text {sym }}$. On the other hand, the continuous measure of symmetry, inherent for pattern " $c$ " quantifying the effort 
necessary for converting this pattern in completely symmetrical is relatively high, namely $\widetilde{\boldsymbol{S}}(\boldsymbol{G})=0.1099(\widehat{\boldsymbol{S}}(\boldsymbol{G})=33.15 \%)$.

Thus, it turns out, that the least effort necessary for the transformation of the polygons in the pattern into the perfectly symmetrical ones is inherent for pattern " $b$ ", the least value of the Voronoi entropy is established for the pattern " $c$ ", the same pattern demonstrates the smallest value of the informational measure of symmetry. It was instructive to define the ratio:

$$
\Psi=\frac{H_{\text {sym }}(G)}{\tilde{S}(G)}
$$

which is supplied in Table 2; it is seen that for the studied Penrose-tiling inspired patterns this ratio is confined within the broad range, namely: $5.18<\Psi<35.7$. This means that the values of the informational and continuous measures of symmetry are not correlated. At the same time the value of the Voronoi entropy $\boldsymbol{S}_{\boldsymbol{v o r}}$ also does not correlate with the informational measure of symmetry $\boldsymbol{H}_{\boldsymbol{s y m}}(\boldsymbol{G})$ and the continuous measure of symmetry $\tilde{\boldsymbol{S}}(\boldsymbol{G})$ (see Table 2 ).

It should be emphasized that the entropy-like mathematically shaped informational measure of symmetry $\boldsymbol{H}_{\text {sym }}(\boldsymbol{G})$, defined by Eqs. 2-3 is the intensive value, describing the patterns generated by the Penrose tiling, in other words, it does not depend on the area of the pattern (when the edge effects are neglected); thus, it is very different from the extensive thermodynamic entropy, as it is discussed in detail in refs. 5-9. On the other hand, it is well expected that IMS will undergo a jump under phasetransitions in quasi-crystals; we plan to study this phase-transitions inspired change in IMS in future. The extension of the IMS to the 3D lattices is straightforward.

\section{Conclusions}

We conclude that quantifying of ordering and quantifying of symmetry in the patterns is a multilayer, perplexed and challenging task. We introduced the informational measure of symmetry $\boldsymbol{H}_{\text {sym }}(G)$ defined as: $\boldsymbol{H}_{\text {sym }}(G)=-\sum_{i=1}^{n} \boldsymbol{P}\left(G_{\boldsymbol{i}}\right) \boldsymbol{l n} \boldsymbol{P}\left(\boldsymbol{G}_{\boldsymbol{i}}\right)$, where $\boldsymbol{P}\left(\boldsymbol{G}_{\boldsymbol{i}}\right)$ is the probability of appearance of the symmetry operation $\boldsymbol{G}_{\boldsymbol{i}}$ within the elements of the given pattern, which is mathematically shaped as the Shannon measure of information [1-2,5-9]. $\boldsymbol{H}_{\text {sym }}(\boldsymbol{G})$ is interpreted as an averaged uncertainty in the presence of symmetry elements from the group $G$ in the given pattern. $\boldsymbol{H}_{\boldsymbol{s y m}}(\boldsymbol{G})$ may also be understood as a measure of the average unexpectedness of presence of symmetry elements constituting group $G$ in the given 2D pattern. Whatever is the interpretation of the informational measure of symmetry $\boldsymbol{H}_{\boldsymbol{s y m}}(\boldsymbol{G})$, it supplies us an averaged information about the entire pattern, and it is not related to the specific symmetry operation $\boldsymbol{G}_{\boldsymbol{i}}$. As an example, the informational measure of symmetry of the "ideal" pattern built of identical equilateral triangles is established as $\boldsymbol{H}_{\text {sym }}\left(\boldsymbol{D}_{3}\right)=1.792$. The informational measure of symmetry of the random, completely disordered pattern is zero. We studied the patterns arising from the Penrose P3 tiling and calculated the Voronoi entropy $\boldsymbol{S}_{\boldsymbol{v} \text { or }}$, the continuous measure of symmetry $\tilde{\boldsymbol{S}}(\boldsymbol{G})$ and the informational measure of symmetry inherent for these patterns. We demonstrated that $\boldsymbol{H}_{\text {sym }}(\boldsymbol{G}), \tilde{\boldsymbol{S}}(\boldsymbol{G})$ and $\boldsymbol{S}_{\boldsymbol{v o r}}$ are not correlated. Our paper reveals the fine structure of quantification of symmetry of patterns: the informational measure of symmetry does not necessarily correlate neither with the effort necessary for symmetrization of the pattern nor with its Voronoi entropy. Thus, quantification of symmetry of the pattern could not be exhausted with the single quantity. It should be emphasized that the introduced informational measure of is the intensive value, describing the patterns generated by the Penrose tiling, in other words, it does not depend on the area of the pattern (when the edge effects are neglected); thus, it is very different from the "true" extensive thermodynamic Boltzmann entropy [5-9].

Author Contributions: Conceptualization, E. B.; I. L and M. F.; methodology, E.B.; M. F.; I. L.; N. S. and S. S; software, I. L., M. F. and N. S.; validation, E.B.; M. F.; I. L.; N. S. and S. S; formal analysis, E.B.; M. F.; I. L.; N. S. and S. S; investigation, I. L. and M. F.; N. S. and S.S.; data curation, E.B.; M. F.; I. L.; N. S. and S. S; writing-original draft preparation, E. B. and M. F.; supervision, E. B. and S. S.; project administration, S. S. All authors have read and agreed to the published version of the manuscript. 
Funding: Edward Bormashenko and Mark Frenkel are thankful for funding to the Russian Science Foundation, Grant number 19-19-00076.

Data Availability Statement: The data that support the findings of this study are available on request from the corresponding author.

Acknowledgments: The authors are thankful to Yelena Bormashenko for her kind help in preparing this paper.

Conflicts of Interest: The authors declare no conflict of interest.

\section{References}

1. Shannon, C. E. A Mathematical Theory of Communication. Bell System Technical Journal, 1948, 27 (3), $379-423$.

2. Shannon, C.E.; Weaver, W. The Mathematical Theory of Communication; The University of Illinois Press: Chicago, IL, USA, 1949.

3. Landau, L. D. ; Lifshitz, E. M. Statistical Physics, 3rd Ed., Course of Theoretical Physics, Vol. 5, Elsevier, Oxford, UK, 2011.

4. Kittel, Ch. Thermal Physics, J. Wiley \& Sons, New York, USA, 1969.

5. Ben-Naim, A. Entropy, Shannon's Measure of Information and Boltzmann's H-Theorem, Entropy 2017, 19(2), 48

6. Ben-Naim, A. Information Theory; World Scientific: Singapore, 2017.

7. Ben-Naim, A. A Farewell to Entropy: Statistical Thermodynamics Based on Information; World Scientific: Singapore, 2008.

8. Ben-Naim, A.; Casadei, D. Modern Thermodynamics; World Scientific: Singapore, 2016.

9. Ben-Naim, A. Entropy and the Second Law. Interpretation and Misss-Interpretationsss; World Scientific: Singapore, 2012.

10. Vstovsky, G.V. Transform information: A symmetry breaking measure. Found. Phys. 1997, 27, 14131444 .

11. Gleiser, M., Stamatopoulos, N. Information content of spontaneous symmetry breaking, Phys. Rev. D 2012, 86, 045004 .

12. Bormashenko, Ed. Entropy, Information, and Symmetry: Ordered is Symmetrical. Entropy 2020, $22,11$.

13. Bormashenko, Ed. Entropy, Information, and Symmetry; Ordered Is Symmetrical, II: System of Spins in the Magnetic Field. Entropy 2020, 22, 235.

14. Zabrodsky, H.; Peleg, S.; Avnir, D. Continuous symmetry measures. J. Am. Chem. Soc. 1992, 114, 78437851. https://doi.org/10.1021/ja00046a033

15. Zabrodsky, H.; Peleg, S.; Avnir, D. Continuous symmetry measures. 2. Symmetry groups and the tetrahedron. J. Am. Chem. Soc. 1993, 115, 8278-8289. https://doi.org/10.1021/ja00071a042

16. Alemany, P., Casanova, D., Alvarez, S., Dryzun, Ch, Avnir D., Continuous Symmetry Measures: A New Tool in Quantum Chemistry, Reviews in Computational Chemistry, Volume 30, ed. by: A. L. Parrill and K. B. Lipkowitz, Wliey, Interscience: Hoboken, NJ, USA, pp. 289-353, 2017

17. Zabrodsky, H.; Avnir, D. Continuous Symmetry Measures. 4. Chirality. J. Am. Chem. Soc. 1995, 117, 462-473. https://doi.org/10.1021/ja00106a053

18. Pinsky, M.; Avnir, D. Continuous Symmetry Measures. 5. The Classical Polyhedra. Inorg. Chem. 1998, 37, 5575-5582. https://doi.org/10.1021/ic9804925

19. Zabrodsky, H.; Peleg, S.; Avnir, D. Symmetry as a continuous feature. IEEE Trans. on Pattern Anal. Mach. Intel. 1995, 17, 1154-1166.

20. Pinsky, M.; Dryzun, Ch.; Casanova, D.; Alemany, P.; Avnir, D. Analytical methods for calculating Continuous Symmetry Measures and the Chirality Measure, Comp. Chemistry 2008, 29, $2712-2721$. https://doi.org/10.1002/jcc.20990

21. Bonjack, M., Avnir, D. The near-symmetry of protein oligomers: NMR-derived structures. Sci Rep 10, 8367 (2020).

22. Sinai, H.E.; Avnir, D. Adsorption-induced Symmetry Distortions in W@Au12 Nanoclusters, Leading to Enhanced Hyperpo-larizabilities, Israel J. Chem. 2016, 56, 1076-1081. https://doi.org/10.1002/ijch.201600082.

23. Bormashenko, Ed; Legchenkova, I.; Frenkel, M.; Shvalb, Shoval, Sh. Voronoi Entropy vs Continuous Measure of Symmetry of the Penrose Tiling: Part I. Analysis of the Voronoi Diagrams, submitted to Entropy, 2021.

24. Voronoi, G. Nouvelles applications des paramètres continus à la théorie des formes quadratiques. Deuxième mémoire. Recherches sur les paralléloèdres primitifs. Reine Angew. Math. 1908, 134, $198-287$. https://doi.org/10.1515/crll.1908.134.198

25. Barthélemy, M. Spatial networks. Phys. Rep. 2011, 499, 1-101. https://doi.org/10.1016/j.physrep.2010.11.002

26. Weaire, D.; Rivier, N. Soap, cells and statistics - random patterns in two dimensions. Contemp. Phys. 1984, 25, 59-99. https://doi.org/10.1080/00107518408210979 
27. Wang S.; Tian, Z.; Dong, K.; Xie, Q. Inconsistency of neighborhood based on Voronoi tessellation and Euclidean distance. J. Alloys Compd. 2021, 854, 156983. https://doi.org/10.1016/j.jallcom.2020.156983

28. Fedorets, A.A.; Frenkel, M.; Shulzinger, E. et al. Self-assembled levitating clusters of water droplets: pattern-formation and stability. Sci. Rep. 2017, 7, 1888. https://doi.org/10.1038/s41598-017-02166-5

29. Liu, YT., Tao, CL., Zhang, X. et al. Mesophasic organization of GABAA receptors in hippocampal inhibitory synapses. Nat. Neurosci. 2020, 23, 1589-1596.

30. Fedorets, A.A.; Frenkel, M.; Bormashenko, E.; Nosonovsky, M. Small Levitating Ordered Droplet Clusters: Stability, Symmetry, and Voronoi Entropy. J. Phys. Chem. Lett. 2017, 8, $5599-5602$. https://doi.org/10.1021/acs.jpclett.7b02657

31. $\mathrm{Xu}, \mathrm{K}$. Geometric formulas of Lewis's law and Aboav-Weaire's law in two dimensions based on ellipse packing, Philosophical Magazine Lett. 2019, 99 (9), 317-325.

32. Frenkel, M.; Arya P.; Bormachenko, Ed.; Santer, S. Quantification of ordering in active light driven colloids, J. Colloid Interface Sci. 2021, 586, 866-875. https://doi.org/10.1016/j.jcis.2020.10.053

33. Bormashenko, Ed.; Frenkel, M.; Vilk, A.; Legchenkova, I.; Fedorets, A.A.; Aktaev, N. E. Dombrovsky, L.A.; Nosonovsky, M. Characterization of self-assembled 2D patterns with Voronoi Entropy. Entropy 2018, 20, 956. https://doi.org/10.3390/e20120956

34. Frenkel, M.; Fedorets, A.A.; Dombrovsky, L.A.; Nosonovsky, M.; Legchenkova, I.; Bormashenko, Ed. Continuous Symmetry Measure vs Voronoi Entropy of Droplet Clusters. J. Phys. Chem. C 2021, 125, $2431-2436$. https://doi.org/10.1021/acs.jpcc.0c10384

35. Bormashenko, E.; Legchenkova, I.; Frenkel, M. Symmetry and Shannon Measure of Ordering. Entropy 2019, 21, 452. https://doi.org/10.3390/e21050452

36. Steinhardt, P., Jeong, HC. A simpler approach to Penrose tiling with implications for quasicrystal formation. Nature, 1996, 382, 431-433 .

37. Shechtman, D.; Blech, I.; Gratias, D.; Cahn, J.W. Metallic phase with long-range orientational order and no translational symmetry. Phys. Rev. Lett. 1984, 53, 1951-1953. https://doi.org/10.1103/PhysRevLett.53.1951.

38. Yaglom, A.M.; Yaglom, I.M. Probability and Information; Jain, V.K., Reidel, D., Eds.; Springer Science \& Business Media: Berlin/Heidelberg, Germany, 1983.

39. Limaye, A.V.; Narhe, R.D.; Dhote, A.M.; Ogale, S.B. Evidence for convective effects in breath figure formation on volatile fluid surfaces. Phys. Rev. Lett. 1996, 76, 3762-3765.

40. Martin, C.P.; Blunt, M.O.; Pauliac-Vaujour, E.; Stannard, A.; Moriarty, P.; Vancea, I.; Thiele, U. Controlling pattern formation in nanoparticle assemblies via directed solvent dewetting. Phys. Rev. Lett. 2007, 99, 116103. 